\title{
Photoluminescence of Bioceramic Materials (PLB) as a Complementary and Alternative Therapy for Diabetes
}

Shoei-Loong Lin ${ }^{1,2}$, Cheuk-Sing Choy ${ }^{3}$, Wing $\mathrm{P}_{\text {Chan }}{ }^{4,5}$ and Ting-Kai Leung ${ }^{5-7 *}$

${ }^{1}$ Department of Surgery, Taipei Hospital, Ministry of health and Welfare, Taiwan

${ }^{2}$ Department of Surgery, School of Medicine, College of Medicine, Taipei Medical University, Taiwan

${ }^{3}$ Emergency and Intensive Care Department, Taipei Hospital, Department of Health, Taiwan

${ }^{4}$ Department of Radiology, School of Medicine, College of Medicine, Taipei Medical University, Taiwan

${ }^{5}$ Department of Radiology, Wan Fang Hospital, Taipei Medical University, Taiwan

${ }^{6}$ Department of Physics, Fu Jen Catholic University, Hsinchuang, Taiwan

${ }^{7}$ Diagnostic Radiology Department, Taipei Hospital, Ministry of Health and Welfare, Taiwan

\begin{abstract}
The rapid rise of diabetes in Asia and Africa is surpassing that of western countries. Diabetes incurs a significant financial burden on patients and national economies. Consequently, factors such as a miserable life-long treatment of hypoglycemic therapy and the possibility of drug intolerance necessitate a search for non-pharmacological alternatives to reduce the requirement of anti-diabetic drugs. Photoluminescence refers to materials that absorb light energy and then release that energy in the form of light. In this study, Photoluminescence of Bioceramic Materials (PLB) was applied to control hyperglycemia and glycosuria in diabetes from the bench to the clinical bedside examination. The PLB treatments resulted in a tendency to promote glucose diffusion into $\mathrm{C} 2 \mathrm{C} 12$ cell line and show a significant decrease in glycosuria in STZ (streptozotocin) induced diabetic rats. The possible mechanisms of the PLB effects on hyperglycemia also correlate with our previous publication and include molecular diffusion, calcium dependent nitric oxide, suppression of oxidative stress and autonomous nervous system regulation. In the future, PLB may have the role of clinical applications on ameliorating hyperglycemia and improving diabetes-related complications.
\end{abstract}

Keywords: Photoluminescence of bioceramic materials; Streptozotocin; Hyperglycemia

\section{Introduction}

The number of people with diabetes mellitus is increasing worldwide, and the area with the greatest potential increase is the AsiaPacific region, especially China where diabetes rates are on the rise due to over-eating and obesity [1]. Diabetes causes a heavy financial burden on patients and national economies. In the United States, the current annual medical costs associated with diabetes mellitus are over US $\$ 98$ billion, including direct and indirect medical costs and lost productivity [2]. In addition, lifetime hypoglycemic therapy and the problem of drug tolerance necessitate the search for non-pharmacological alternative therapies. There is evidence that an increasing number of people in the United States use one or more alternative remedies for the treatment of common chronic diseases, such as diabetes mellitus. A previous study showed that patients with diabetes mellitus and other common chronic medical conditions were more likely to use alternative medicine than people in the general population. One of the reasons is inadequacies in current treatments for diabetes, which probably led several millions of Americans to use alternative medicine for diabetes treatment, despite the fact that the safety and efficacy of standard medical treatments were proved in limited studies [3]. A recent survey of diabetes educators in the western United States found that the most frequently recommended and used alternative therapies among diabetic patients included nutritional methods, spiritual healing, herbal remedies, massage, meditation, physical activity, acupuncture, homeopathy, relaxation therapy, and music therapy [4-6].

Previous studies have shown that photoluminescence of Bioceramic materials (PLB) treatment promote microcirculation in patients [7] and the up-regulation of calcium-dependent nitric oxide and calmodulin in cell lines $[8,9]$. We previously demonstrated that PLB treatment promotes nitric oxide enhancement through calcium-dependent nitric oxide synthetase $[8,9]$. The PLB treatment demonstrated antioxidant effects by increasing hydrogen peroxide scavenging processes in RAW264.7 murine macrophages [10], MC3T3-E1 murine calvariaderived osteoblast-like cells [11,12], NIH3T3 fibroblasts [13], and C2C12 murine myoblasts [14] and other cell and animal experimental effects [15-18]. The PLB treatment may contribute to physical, chemical and biological properties on different testing specimens, such as water, volatile material, weak acid and living cells, as well as enhancement of transdermal delivery of different drugs [19-21]. According to a series of our human trials by application of BIOCERAMIC material made, we found reduced muscle stiffness of neck as well as musculoskeletal disorders from extended use of computers and overwork. PLB has a tendency to stimulate parasympathetic responses, which may reduce pain of dysmenorrhea, resting energy expenditure and improve cardiorespiratory recovery following exercise, as well as induce flowwave effect on acupuncture points through meridians [22-26]. This study presents a series of physical-biological experiments on the material based on its characteristics of biological effects of electromagnetic non-ionizing radiation [27], nonlinear photonic crystal [28], and photoluminescence $[29,30]$. We study the effects of Bioceramic that emits high-performance far-infrared rays, with combined visible

*Corresponding author: Ting Kai Leung, Department of Radiology, Taipe Hospital, Ministry of Health and Welfare, NO.127, Su Yuan Road, Hsin Chuang District, New Taipei City, 242-13, Taiwan, R.O.C., Tel: 886-2-22765566+2187; Fax: 886-2- 22765591; E-mail: hk8648@tmu.edu.tw

Received November 18, 2013; Accepted December 26, 2013; Published December 30, 2013

Citation: Lin SL, Choy CS, Chan WP, Leung TK (2013) Photoluminescence of Bioceramic Materials (PLB) as a Complementary and Alternative Therapy for Diabetes. J Diabetes Metab 4: 321. doi:10.4172/2155-6156.1000321

Copyright: $\odot 2013$ Lin SL, et al. This is an open-access article distributed unde the terms of the Creative Commons Attribution License, which permits unrestricted use, distribution, and reproduction in any medium, provided the original author and source are credited. 
Citation: Lin SL, Choy CS, Chan WP, Leung TK (2013) Photoluminescence of Bioceramic Materials (PLB) as a Complementary and Alternative Therapy for Diabetes. J Diabetes Metab 4: 321. doi:10.4172/2155-6156.1000321

light spectrum, under the concept of 'Photoluminescence effect'. We evaluated a new innovative application of PLB treatment as a complementary method to control hyperglycemia and glycosuria in diabetes patients by using a cell model and an animal model. To our knowledge, this is the first non-conventional complementary therapy with a focus on 'Photoluminescence effect' that shows beneficial effects in diabetes.

\section{Materials and Methods}

\section{Bioceramic powder}

The ceramic powder was obtained from the Department of Radiology, Taipei Medical University Hospital (Taipei, Taiwan). The Bioceramic material consisted of micro-sized particles produced primarily from different natural elemental components. The elemental analysis of the Bioceramic powder using electron microscopy equipment with electron beam processing on the selected spectrum lists: Calcium (Ca), Zirconium (Zr), Sulphur (S), Silicon(Si), Aluminum $(\mathrm{Al})$, Magnesium $(\mathrm{Mg})$, Iron $(\mathrm{Fe})$, oxygen $(\mathrm{O})$ and Carbon [20].

\section{Photoluminescence of bioceramic materials (PLB)}

Photoluminescence is a special type of luminescence. The term photoluminescence refers to any material that absorbs light energy and then releases that energy in the form of light, and concerns the interaction between electromagnetic radiation and matter. PLB uses a visible red-light source of light-emitting diodes (LEDs), which emit a wavelength of a visible red light spectrum between 620 and $750 \mathrm{~nm}$.
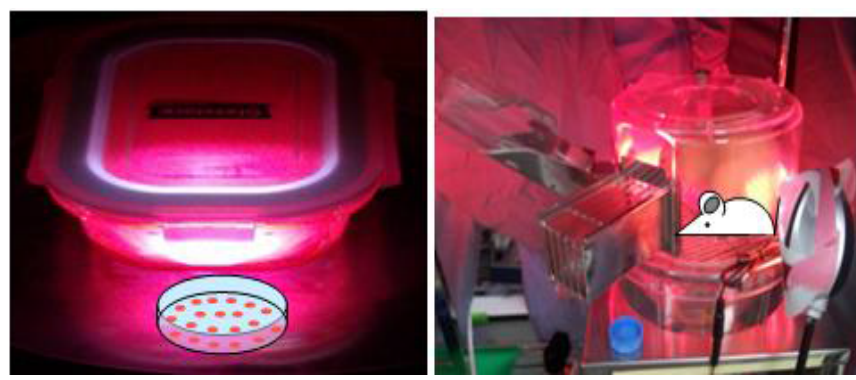

Figure 1: The PLB irradiation procedure used in the cell culture experiments (1a: left upper); The PLB irradiation of rats with STZ-induced diabetes in metabolic cages (1b: right upper).
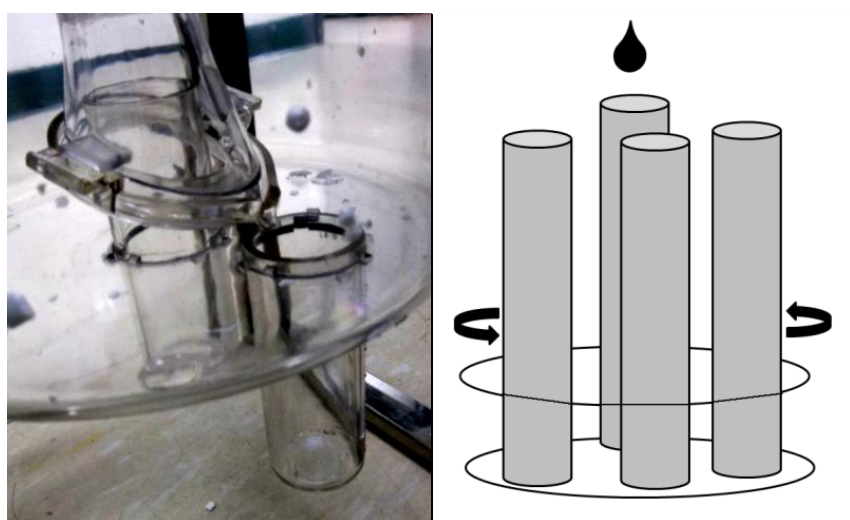

Figure 2: A metabolic cage system with fecal pellet or the urine to flow down into separate collection tubes (2a, right); An innovative design of our autonomic spinning collecting system for urine drop collection, at a speed of one circle per day ( $2 \mathrm{~b}$ left). Thus, four urine tubes could be collected at about 6 hour-interval.
The visible red light spectrum has the characteristics of deep optical penetration depth [31]. We also strictly controlled the light illumination at $450 \pm 50$ lux, without additional thermal effect on cells, animals and human beings.

\section{Cell culture experiments}

The PLB treatment was evaluated using murine myoblast cell line (C2C12, (BCRC, Taiwan). The C2C12 cells were cultured in DMEM (Dulbecco's modified Eagle's medium) containing 10\% fetal bovine serum at $37^{\circ} \mathrm{C}$ in $5 \% \mathrm{CO}_{2}$ in a humidified incubator. The cells were seeded in 12-well plates, and grown for $24 \mathrm{~h}$ to $50 \%$ confluence. The medium was replaced with DMEM containing $10 \%$ horse serum to allow cell differentiation for 5 days. The medium was replaced with 0.5 mL MEM (Eagle minimum essential medium) containing 10\% serum with or without $300 \mathrm{nM}$ insulin. Two groups of control and far-infrared ray (FIR) treatment plates were placed in metal containers, and the PLB source was placed under the FIR group plates (Figures 1a and 1b). At $0,1,2,3,4$, and $5 \mathrm{~h}, 10 \mu \mathrm{L}$ of the medium was withdrawn, and mixed with $1 \mathrm{~mL}$ of a Glucose Kit reagent (Randox, UK) for $30 \mathrm{~min}$. Absorbance at $500 \mathrm{~nm}$ was measured using a spectrophotometric plate reader (OD500). The concentration of glucose in the media was directly proportional to the absorbance.

\section{Animal model experiments}

Sprague-Dawley rats $(180 \pm 10 \mathrm{~g})$ were maintained in cages at $23^{\circ} \mathrm{C}$ $\pm 2^{\circ} \mathrm{C}$ and $60 \% \pm 5 \%$ humidity with 12 -h light/dark cycling. Solid rat food and water were continuously available during the first week to allow the rats to adjust to the environment. The animal experiments were performed during the subsequent $3 \mathrm{wk}$ period. All procedures were conducted in accordance with the ethical guidelines for animal experiments established by our institution (IACV CApproval No: LAC101-0093). Urine glucose levels were assessed immediately using an enzyme assay kit (Randox Laboratories, Antrim, UK), and the STZtreated rats were identified as diabetes based on urine glucose levels above $2.5 \mathrm{~mol} / \mathrm{L}$ [32]. Usually, the non-treated diabetic rats were further confirmed by a progressive increase in urinary glucose. Non-invasive measurement of urinary glucose indirectly indicates the status of hyperglycemia (blood glucose $>30 \pm 2 \mathrm{mmol} / \mathrm{L}$ ) without the physical or psychological damage associated with blood sampling in rats [33]. We were using metabolic cages, which were designed to effectively separate feces and urine into separate tubes outside the cage. The feces and urine collection tubes were made of non-wetting polymethylpentene and allowed the fecal pellet or the urine to flow down into separate collection tubes. The cages were constructed in such a manner that the urine never washes over into the feces tube (Figure 2a) Thus, the separation was immediate and complete, with no cross-contamination of urine and feces. We also designed an innovative automatic spinning platform to collect urine for each sample at 6 hour- intervals. As we decide to perform this experiment on different groups of rats in the same time, in order to omit the possible unpredicted error due to different environmental factors. We used our special design metabolic cage equip with autonomic spinning collecting platform (Figure $2 b$ ). The design is to guarantee the urine collection as frequent as possible, so as to decrease proportion of samples lost error that may be drip off accidentally. We set the spinning speed at one circle per day. Thus, four urine tubes could be collected at about 6 hour-interval. The animals were divided into a control group and PLB groups. Ten rats were divided into health group (two rats), diabetic non-PLB group (two rats) and diabetic PLB group (six rats). Diabetic groups of rats that had undergone a $1 \mathrm{wk}$ adjustment period for diabetes induction which were fasted for at least 12 hours, and $60 \mathrm{mg} / \mathrm{kg}$ streptozotocin (STZ) in a 0.01 
Citation: Lin SL, Choy CS, Chan WP, Leung TK (2013) Photoluminescence of Bioceramic Materials (PLB) as a Complementary and Alternative Therapy for Diabetes. J Diabetes Metab 4: 321. doi:10.4172/2155-6156.1000321

Page 3 of 6

(a)

C2C12 cell - PLB test

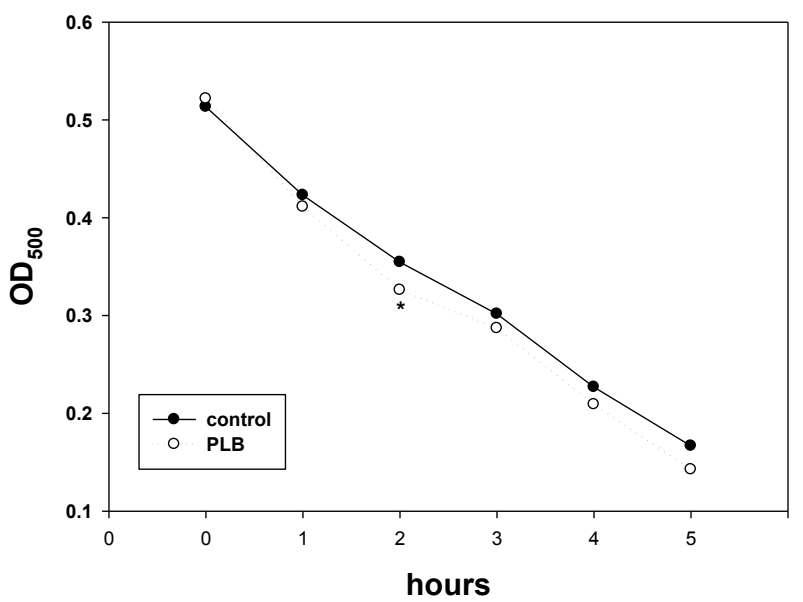

(b)

C2C12 cell - PLB test (no insulin)

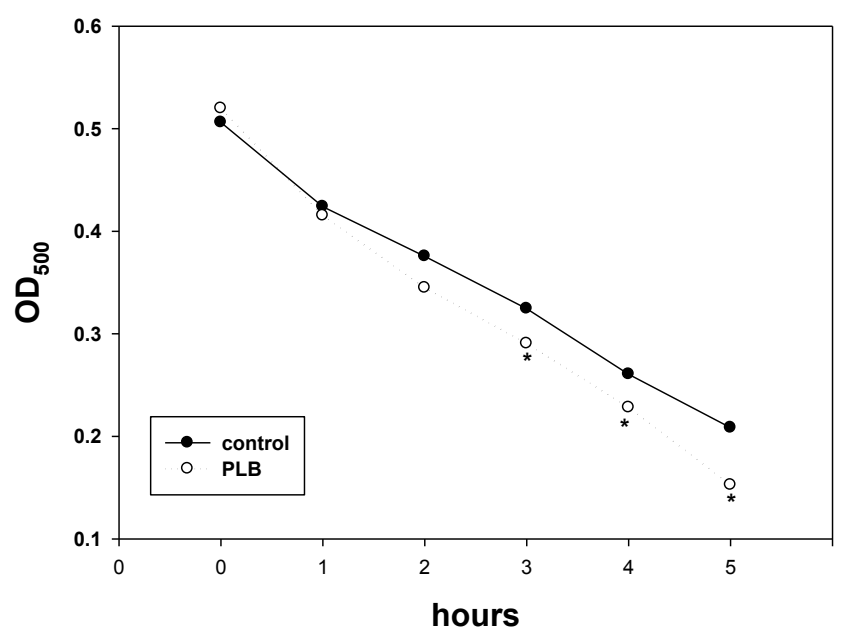

Figure 3: The effect of PLB irradiation on the uptake of glucose by differentiated $\mathrm{C} 2 \mathrm{C} 12$ cells with (a) and without (b) insulin treatment. The concentration of glucose in the cell culture medium was assessed by absorbance at $500 \mathrm{~nm}$. ${ }^{*} p<0.05$ when compared with the control.

M citrate buffer was injected intraperitoneally. An equivalent volume of normal saline was used for the peritoneal injection for rats in the health group. About 5 days after STZ injection, base on the glucosuria levels, diabetic PLB group were subdivided into high glucosuria subgroup (two rats) (urine glucose levels above $2.5 \mathrm{~mol} / \mathrm{L}$ ), moderate glucosuria subgroup (two rats) (urine glucose levels almost equals to $2.5 \mathrm{~mol} / \mathrm{L}$ ) and low glucosuria subgroup (two rats) (urine glucose levels less than $2.5 \mathrm{~mol} / \mathrm{L})$. The diabetic PLB sub-groups received PLB treatment $(4 \mathrm{~h} / \mathrm{d})$ for continuously $5 \mathrm{~d}$ in a $1 \mathrm{wk}$ period (Figure $1 \mathrm{~b}$ ). The light sources were kept at $10 \mathrm{~cm}$ away from the metabolic cage, to maintain a constant light illumination without significantly thermal effect on the rats.

\section{Data analysis}

Statistical analyses were performed using SPSS 15.0 software (SPSS Inc, Chicago, Ill, USA). We examined the individual variables by using means and standard error, exploring group differences by using oneway ANOVA analysis (Design Expert, Stat-Ease Inc, USA).

\section{Results}

\section{PLB treatment increases glucose uptake in cell culture}

The analysis of the culture media showed decreases in glucose concentration with and without insulin treatment; meanwhile the PLB treatment of $\mathrm{C} 2 \mathrm{C} 12$ cell culture in both cases show the significantly better glucose uptake by cells in comparison with the control groups (Figures $3 \mathrm{a}$ and $3 \mathrm{~b}$ ). Although only one point ( $2 \mathrm{~h}$ of PLB treatment in Figure $3 a$ ) shows the significant difference ( $p$-value $<0.05$ ) of measured glucose concentration in cell culture containing insulin, most of the cell culture without insulin show the significant difference of measured glucose concentration after PLB treatment (Figure 3b).

\section{PLB treatment reduces urinary glucose in STZ-induced diabetic rats}

The autonomic spinning platform for urine collection was running smoothly, with urine sample lost proportion less than $3 \%$. It was taken about 25 days to finish the samples collection with maximum sample number as ' 97 ' on each sub-groups diabetic rats. Similar to our initial prediction, there was no significant increase of glucosuria in healthy group of rats (Figure 4). The diabetes non-PLB group is also progressive increase of their glucosuria during the examination. The fluctuation range of glucosuria in diabetes non-PLB group was not lower than $2.6 \mathrm{~mol} / \mathrm{L}$ (Figure 4). On the contrary, Table 1 and Figure 4 show that there are trends of decreased urinary glucose on diabetic rats with PLB treatment; it reflects the amelioration of hyperglycemia. Besides, the different subgroups of diabetes PLB group demonstrated different degrees of amelioration on their glucosuria. Table 1 shows significant differences ( $p$-value $<0.05$ ) of measured urine glucose between diabetic rats without PLB (control group), and with PLB treatment (H, M, and $\mathrm{L}$ groups). Besides, there are significant differences of measured urine glucose between diabetic rats of different degree of glucosuria $(\mathrm{H}$, $\mathrm{M}$, and $\mathrm{L}$ groups). Base on the results of Table 1, the improvements of glucosuria are in descending order as: low glucosuria subgroup $>$ moderate glucosuria subgroup $>$ high glucosuria subgroup.

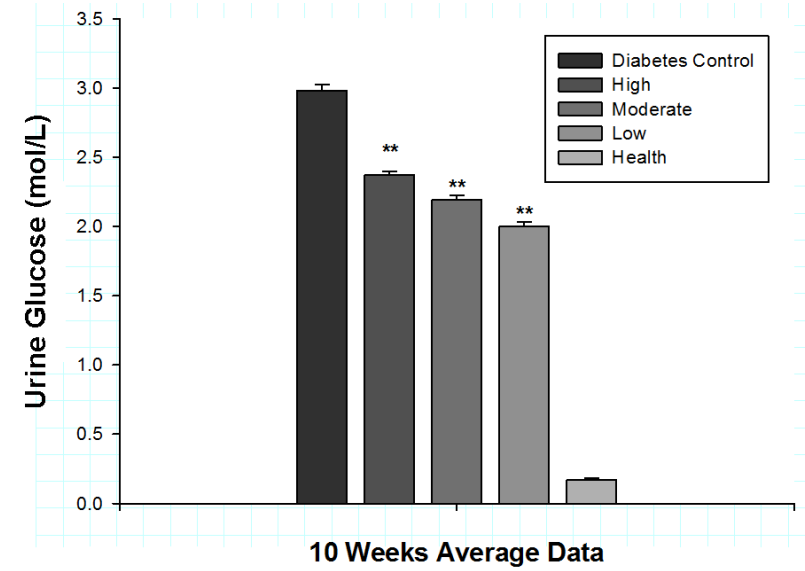

Figure 4: STZ-induced diabetic rats were the highest amount of glucosuria, while healthy rats were approach zero of urine sugar. The effect of PLB treatment on the urinary glucose levels in STZ-induced diabetic rats (subdivided as high $(\mathrm{H})$, moderate $(\mathrm{M})$, and low $(\mathrm{L})$ glucosuria subgroups). The rats received PLB treatment for $4 \mathrm{~h} / \mathrm{d}$ over a 5 -d period. Urine glucose levels were assessed immediately using an enzyme assay kit (Randox Laboratories, Antrim, UK). ${ }^{* *} \mathrm{p}<0.05$ when compared with the control. 
Citation: Lin SL, Choy CS, Chan WP, Leung TK (2013) Photoluminescence of Bioceramic Materials (PLB) as a Complementary and Alternative Therapy for Diabetes. J Diabetes Metab 4: 321. doi:10.4172/2155-6156.1000321

Page 4 of 6

\begin{tabular}{|c|c|c|c|}
\hline Groups (observations) & Estimated mean urine glucose & Standard error & \\
\hline 1-control $(n=38)$ & 2.98 & 0.052 & \\
\hline $2-H(P L B)(n=97)$ & 2.43 & 0.032 & \\
\hline 3-M (PLB) $(n=97)$ & 2.28 & 0.032 & \\
\hline 4-L (PLB) (n=97) & 2.11 & 0.032 & \\
\hline 5-health $(n=62)$ & 0.17 & 0.040 & \\
\hline Treatment & Mean difference & Standard error & P-value \\
\hline 1 vs. 2 & 0.55 & 0.061 & $<0.0001$ \\
\hline 1 vs. 3 & 0.70 & 0.061 & $<0.0001$ \\
\hline 1 vs. 4 & 0.87 & 0.061 & $<0.0001$ \\
\hline 1 vs. 5 & 2.81 & 0.066 & $<0.0001$ \\
\hline 2 vs. 3 & 0.15 & 0.046 & 0.0010 \\
\hline 2 vs. 4 & 0.32 & 0.046 & $<0.0001$ \\
\hline 2 vs. 5 & 2.26 & 0.052 & $<0.0001$ \\
\hline 3 vs. 4 & 0.17 & 0.046 & 0.0002 \\
\hline 3 vs. 5 & 2.11 & 0.052 & $<0.0001$ \\
\hline 4 vs. 5 & 1.94 & 0.052 & $<0.0001$ \\
\hline
\end{tabular}

Note: control is diabetic rats without PLB treatment; $\mathrm{H}, \mathrm{M}$, and $\mathrm{L}$ groups are high, moderate, and low glucosuria of diabetic rates, respectively with PLB treatment; health group is healthy rats. Observation is number of measured urine glucose from two rats in each group within 10 weeks of experimental period. P-value $<0.05$ means significant difference of comparison of two groups.

Table 1: Comparision of mean urine glucose $(\mathrm{mol} / \mathrm{L})$ of rats with and without PLB treatment.

\begin{tabular}{|c|c|c|}
$\begin{array}{c}\text { Hypothesis base on previous publications } \\
\text { of 'Photoluminescence of Bioceramic } \\
\text { Materials'(PLB) }\end{array}$ & Possible mechanism of PLB to reduce hyperglycemia \\
\hline Molecular diffusion & $\begin{array}{c}\text { Through weaken the hydrogen bonds between water molecules. Thus, the changes } \\
\text { in the physical properties of water may promote the diffusion of glucose across cell } \\
\text { membrane into intracellular space }\end{array}$ \\
\hline Calcium dependent nitric oxide enhancement & $\begin{array}{c}\text { Bioceramic irradiation promotes nitric oxide (NO) enhancement through calcium- } \\
\text { dependent nitric oxide synthetase, which is beneficial for glucose metabolism. }\end{array}$ \\
\hline Suppression of oxidative stress & $\begin{array}{c}\text { Oxidative stress lead to abnormal changes in intracellular signaling and result } \\
\text { in chronic inflammation and insulin resistance, and then increase risk of } \\
\text { hyperglycemia. PLB was proved to suppress oxidative stress. }\end{array}$ \\
\hline [8, 9]
\end{tabular}

Table 2: Hypothesis and mechanism of PLB's effect on hyperglycemia.

\section{Extracellular space}

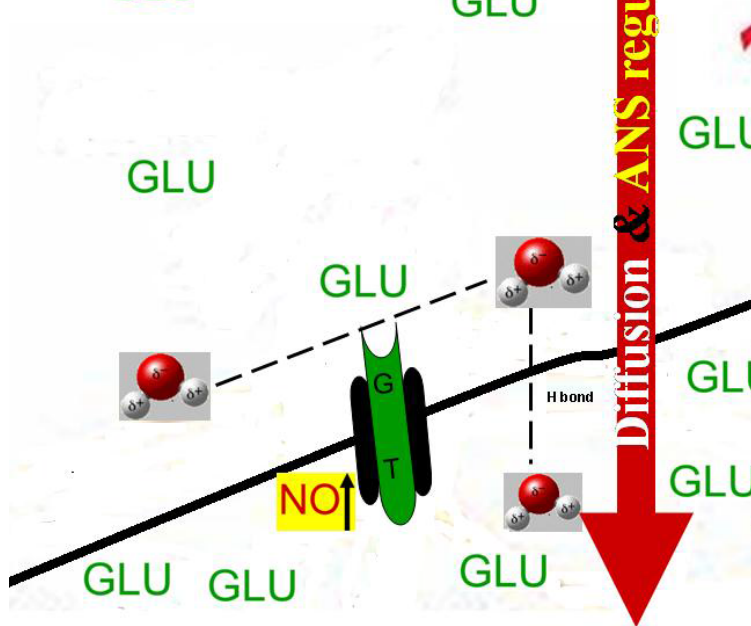

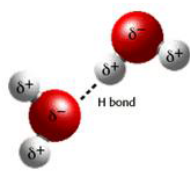

GLU

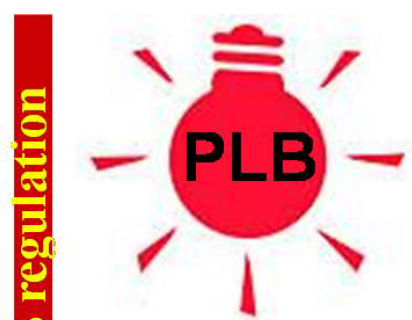

GLU
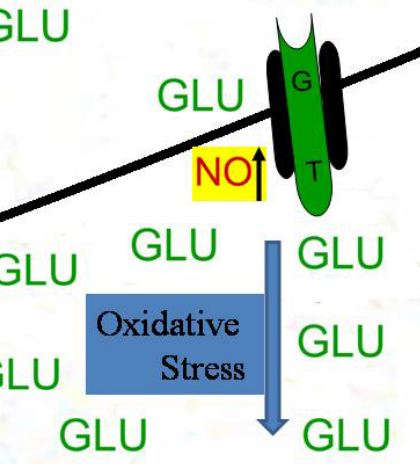

Intracellular space

Figure 5: The possible mechanisms of PLB treatment to increase glucose (GLU) transportation from extracellular to intracellular space are include molecular diffusion, calcium dependent nitric oxide, suppression of oxidative stress and autonomous nervous system regulation 


\section{Discussion}

The PLB treatments resulted in a tendency of promote glucose diffusion into $\mathrm{C} 2 \mathrm{C} 12$ cell line (Figure 3 ) and a significant decrease of glucosuria in STZ induced diabetic rats (Figure 4). We found that moderate or low glucosuria subgroup of diabetic rats, had better outcome by using PLB treatment. This finding was also observed in our preliminarily unreleased data which showed that patients with fasting blood glucose levels below $200 \mathrm{mg} / \mathrm{mL}$ had better outcomes by using PLB treatment, compared to fasting blood glucose level higher than 200 $\mathrm{mg} / \mathrm{mL}$. The possible mechanisms of the PLB effects on hyperglycemia should be combined with the present results and correlated with our previous published data [7-26]. They include molecular diffusion, calcium dependent nitric oxide, suppression of oxidative stress and autonomous nervous system regulation (Table 2 and Figure 5).

The emission wavelengths of Bioceramic materials can weaken the hydrogen bonds between water molecules. Thus, the changes in the physical properties of water may promote the diffusion of solutes, improving the transport of substances across the cell membrane from the blood into cells [19-21]. Therefore, the increased uptake of glucose by the cell culture after PLB treatment (Figures 2a and 2b) may be due to the increased permeability of glucose through the cell membrane. Our previous results found that Bioceramic irradiation promotes nitric oxide (NO) enhancement through calcium-dependent nitric oxide synthetase $[8,9]$. By the help of additional visible light spectrum on Bioceramic materials, the PLB treatment process would augment more calcium-dependent nitric oxide production [8,9]. And, as we know, nitric oxide is beneficial for diabetics for glucose metabolism [34-36]. It was reported that the large increase in blood glucose utilization by skeletal muscle is mediated by the translocation of the glucose transporter from an intracellular pool to the sarcolemma of skeletal muscle myocytes of rodents [35,36]. Nitric oxide mediates glucose uptake into skeletal muscle during exercise by affecting glucose transporter translocation. Nitric oxide donors, such as sodium nitroprusside (SNP), cause a dose dependent increase in skeletal muscle glucose transport [34-36], maintaining the perfusion of the myocardium with normal levels of blood glucose. The nitric oxide and cyclic guanosine monophosphate system (NO/cGMP) is the insulinindependent and contraction-independent pathway for stimulating muscle glucose transport by increasing cell surface glucose transporter protein [36-43]. Nitric Oxide also plays a significant role as a second messenger of insulin mediated signals [44]. Thus, PLB irradiation to enhanced calcium dependent nitric oxide production is another possible mechanism to reduce blood sugar level of hyperglycemia. As mentioned before, PLB was proved to suppress oxidative stress by hydrogen peroxide [10-14]. Increased production of hydrogen peroxide or reduced capacity for hydrogen peroxide elimination, termed oxidative stress, can lead to abnormal changes in intracellular signaling. Oxidative stress is then causing chronic inflammation of pancreatic $\beta$ cells, and then dysfunction of the cells is a potential mechanism causing impaired glucose-induced insulin secretion. Oxidative stress may also be related to insulin resistance, and then increase risk of hyperglycemia $[45,46]$. According to a series of our human trials by using Heart Rate Variability (HRV) test, it was found that PLB helps regulate the autonomous nervous system and enhance parasympathetic functioning [22-24]. In brief, the sympathetic and parasympathetic branches are the two components of the Autonomic Nervous System (ANS). Just as the sympathetic nervous system controls of the body's internal organs to deal with stress, as in the flight-or-fight response, its counter-balancing system-the parasympathetic system-dominates to maintain a condition of rest and repair. Stimulation of sympathetic nervous system relates to the epinephrine level released from the adrenal medulla, which acts to promote release of glucose from glycogen and enhances release of fatty acids from adipose tissue, and results in blood sugar elevation. It was found that excess stimulation by the sympathetic nervous system activity is associated with hyperglycemia or even onset of type II diabetes. PLB's particular effect on the parasympathetic system helps to better balance the ANS, and by reducing the activity of the sympathetic system, PLB would therefore be useful in modulating hyperglycemia [47].

PLB may have the role of clinical applications on ameliorating hyperglycemia and improving diabetes-related complications, such as the restoration of normal myocardial perfusion and other vascular diseases. In this study, we accomplished using our innovative autonomic spinning platform design without disturbing the rats' major physiological conditions. Base on the spirit of "do no harm," we also obtained sample collections without using any invasive blood sample collection technique. Compared with the previous similar studies, as far as we know, the design of this study has an advantage of collecting large number urine samples-an average of 4 urine sample per day from each rat. But, as mentioned, this experiment is limited by a small scale of cell and animal number. The study is also limited by difficulty to show a curve of ameliorative effects on hyperglycemia after PLB treatment. The fact remains that the average urine glucose change is significantly decreased on each diabetic subgroups after PLB treatment. Since urine glucose is passively reflected in actual blood sugar levels, we found the urine glucose change is not always synchronous on the same group or subgroup of rats. Thus, we cannot show a precise timing curve of glucoselowering effect of PLB from our results. We hope that the stepping up investigations may help us to explore more precise mechanism of PLB on hyperglycemia and collect more clinical data and experiences. This study is also constricted by lacking interchangeable method to receive the minimal required exposure time and PLB intensity, to obtain an equivalent biological effect on cells, animal, and human patients. Since there are differences among the above models, the total cell/body surface area, skin thickness and other different factors of in-vitro and in-vivo environments, further fundamental researches on physicalbiological factors are required. Before this promising non-invasive and non-ionized radiation method of PLB treatment can become a mainstream medical application, different fields of cooperation are required. To achieve better therapeutic results, future studies of PLB devices should provide higher levels of irradiation over larger surface areas, for longer exposure periods.

\section{Acknowledgment}

The authors gratefully acknowledge the support provided to this study by Project fund of Taipei Medical University (Project No.:TMU101-AE1-B36) and the contributions of Mimmo Gasbarri, Hen-Yu Liu and C.-Will Chen.

\section{References}

1. Xin Z, Liu C, Niu WY, Feng JP, Zhao L, et al. (2012) Identifying obesity indicators which best correlate with type 2 diabetes in a Chinese population. BMC Public Health 12: 732.

2. American Diabetes Association (2013) Economic costs of diabetes in the U.S in 2012. Diabetes Care 36: 1033-1046.

3. Dham S, Shah V, Hirsch S, Banerji MA (2006) The role of complementary and alternative medicine in diabetes. Curr Diab Rep 6: 251-258.

4. Elder NC, Gillcrist A, Minz R (1997) Use of alternative health care by family practice patients. Arch Fam Med 6: 181-184.

5. Burg MA, Hatch RL, Neims AH (1998) Lifetime use of alternative therapy: a study of Florida residents. South Med J 91: 1126-1131.

6. McWhorter LS (2001) Biological Complementary Therapies: A Focus on Botanical Products in Diabetes. Diabetes Spectrum 14: 199-208. 
Citation: Lin SL, Choy CS, Chan WP, Leung TK (2013) Photoluminescence of Bioceramic Materials (PLB) as a Complementary and Alternative Therapy for Diabetes. J Diabetes Metab 4: 321. doi:10.4172/2155-6156.1000321

7. Lin YS, Lin MY, Leung TK, Liao CH, Huang TT, et al. (2007) Properties and biological effects of high performance ceramic powder emitting far-infrared irradiation. Instrum Today 6: 60-66.

8. Leung TK, Lee CM, Lin MY, Ho YS, Chen CS, et al. (2009) Far infrared ray irradiation induces intracellular generation of nitric oxide in breast cancer cells. J Med Biol Eng 29: 15-18.

9. Leung TK, Lin YS, Chen YC, Shang HF, Lee YH, et al. (2009) Immunomodulatory effects of far infrared ray irradiation via increasing calmodulin and nitric oxide production in RAW 264.7 macrophages. Biomed Eng Appl Basis 21: 317-323.

10. Leung TK, Lin YS, Lee CM, Chen YC, Shang HF, et al. (2011) Direct and indirect effects of ceramic far infrared radiation on hydrogen peroxide-scavenging capacity and on murine macrophages under oxidative stress. J Med Biol Eng 31: $345-351$

11. Leung TK, Shang HF, Chen DC, Chen JY, Chang TM, et al. (2011) Effects of far infrared rays on hydrogen peroxide-scavenging capacity. Biomed Eng App Basis 23: 99-105.

12. Leung TK, Chen $\mathrm{CH}$, Lai $\mathrm{CH}$, Lee CM, Chen CC, et al. (2012) Bone and joint protection ability of ceramic material with biological effects. Chin J Physiol 55: $47-54$

13. Leung TK, Lee CM, Tsai SY, Chen YC, Chao JS (2011) A Pilot Study of Ceramic Powder Far-Infrared Ray Irradiation (cFIR) on Physiology: Observation of Cell Cultures and Amphibian Skeletal Muscle. Chin J Physiol 54: 247-254.

14. Leung TK, Chen CH, Tsai SY, Hsiao G, Lee CM (2012) Effects of far infrared rays irradiated from ceramic material (BIOCERAMIC) on psychological stress-conditioned elevated heart rate, blood pressure, and oxidative stresssuppressed cardiac contractility. Chin J Physiol 55: 323-330.

15. Leung TK, Chan CF, Lai PS, Yang CH, Hsu CY, et al. (2012) Inhibitory effects of far-infrared irradiation generated by ceramic material on murine melanoma cell growth. Int J Photoenergy.

16. Leung TK, Lee CM, Wu CH, Chiou JF, Huang PJ, et al. (2013) The protective effect of non-ionized radiation from far infrared ray emitting ceramic material (cFIR) against oxidative stress on human breast epithelial cell (MCF-10A). J Med Biol Eng.

17. Leung TK, Liu YC, Chen CH, Fang HN, Chen KC, et al. (2013) In vitro cell study of the possible anti-inflammatory and pain relief mechanism of far-infrared ray emitting ceramic material (BIOCERAMIC). J Med Biol Eng 33: 179-184.

18. Leung TK, JM Lin, Chien HS, Day TC (2012) Biological effects of melt spinning fabrics composed of 1\% bioceramic material. Text Res J 83: 1121-1130.

19. Leung TK, Huang PJ, Chen YC, Lee CM (2011) Physical chemical test platform for room temperature, far-infrared ray emitting ceramic materials (cFIR). J Chin Chem Soc 58: 653-658

20. Leung TK, Yang JC, Lin YS (2012) The physical, chemical and biological effects by room temperature ceramic far-infrared ray emitting material irradiated water: a pilot study. J Chin Chem Soc 58: 1-6.

21. Lin SL, Chan WP, Choy CS, Leung TK (2013) Enhancement of Transderma Delivery of Indomethacin and Tamoxifen by Far-Infrared Ray-Emitting Ceramic Material (BIOCERAMIC): A Pilot Study TransI Med 3: 1

22. Lai CH, Leung TK, Peng CW, Chang KH, Lai MJ, et al. (2013) Effects of FarInfrared Irradiation on Myofascial Neck Pain: A Randomized, Double-Blind, Placebo-Controlled Pilot Study. J Altern Complement Med.

23. Lin SL, Chan WP, Choy CS, Leung TK (2013) Translating laboratory research of BIOCERAMIC material, application on computer mouse and bracelet, to ameliorate computer work-related musculoskeletal disorders. Translational Medicine.

24. Liau BY, Leung TK, Ou MC, Ho CK, Lin YS (2012) Inhibitory Effects of farinfrared ray generating belt on primary dysmenorrheal. Int. J. Photoenergy.

25. LeungTK, Kuo CH, Lee CM, Kan NW, Hou CW (2013) Physiological effects of bioceramic material: Harvard Step, Resting Metabolic Rate and Treadmill Running Assessments. Chin J Physiol 56: 1-7.

26. Chen CW, Tai CJ, Choy CS, Hsu CY, Lin SL, et al. (2013) Wave-induced flow in meridians demonstrated using photoluminescent bioceramic material on acupuncture points. Evid Based Complement Alternat Med 2013: 739293.

27. Velizarov S, Raskmark $P$, Kwee S (1999) The effects of radiofrequency fields on cell proliferation are non-thermal. Bioelectrochem Bioenerg 48: 177-180.

28. Tkeshelashvili L (2003) Nonlinear photonic crystal asers and Electro-Optics Europe. CLEO/Europe.
29. Gilliland GD (1997) Photoluminescence spectroscopy of crystalline semiconductors. Materials Science and Engineering R 18: 99-400.

30. Yu H, Lycett S, Roberts C, Murray R (1996) Time resolved study of selfassembled InAs quantum dots. Applied Physics Letters 69: 4087-4089.

31. Holder MJ, Milward MR, Palin WM, Hadis MA, Cooper PR (2008) Lasers and lights---a practical update and future considerations. Light-Emitting Diodes (LEDs) in dermatology daniel barolet. Surgery 27: x227-238.

32. Kumar C, Rachappaji K, Nandini C, Sambaiah K, Salimath P (2002) Modulatory effect of butyric acid-a product of dietary fiber fermentation in experimentally induced diabetic rats. J Nutr Biochem 13: 522

33. Harris MI, Hadden WC, Knowler WC, Bennett PH (1987) Prevalence of diabetes and impaired glucose tolerance and plasma glucose levels in U.S population aged 20-74 yr. Diabetes 36: 523-534.

34. Balon TW, Nadler JL (1997) Evidence that nitric oxide increases glucose transport in skeletal muscle. J Appl Physiol (1985) 82: 359-363.

35. Etgen GJ Jr, Fryburg DA, Gibbs EM (1997) Nitric oxide stimulates skeletal muscle glucose transport through a calcium/contraction- and phosphatidylinositol-3kinase-independent pathway. Diabetes 46: 1915-1919.

36. Young ME, Radda GK, Leighton B (1997) Nitric oxide stimulates glucose transport and metabolism in rat skeletal muscle in vitro. Biochem J 322: 223 228.

37. Kersten JR, Toller WG, Tessmer JP, Pagel PS, Warltier DC (2001) Hyperglycemia reduces coronary collateral blood flow through a nitric oxidemediated mechanism. Am J Physiol Heart Circ Physiol 281: H2097-H2104.

38. Scherrer U, Randin D, Vollenweider P, Vollenweider L, Nicod P (1994) Nitric oxide release accounts for insulin's vascular effects in humans. J Clin Invest 94: $2511-2515$

39. Steinberg HO, Brechtel G, Johnson A, Fineberg N, Baron AD (1994) Insulinmediated skeletal muscle vasodilation is nitric oxide dependent. A novel action of insulin to increase nitric oxide release. J Clin Invest 94: 1172-1179.

40. Ting HH, Timimi FK, Boles KS, Creager SJ, Ganz P, et al. (1996) Vitamin C improves endothelium-dependent vasodilation in patients with non-insulindependent diabetes mellitus. J Clin Invest 97: 22-28.

41. Du XL, Edelstein D, Dimmeler S, Ju Q, Sui C, et al. (2001) Hyperglycemia inhibits endothelial nitric oxide synthase activity by posttranslational modification at the Akt site. J Clin Invest 108: 1341-1348.

42. Bradley SJ, Kingwell BA, McConell GK (1999) Nitric oxide synthase inhibition reduces leg glucose uptake but not blood flow during dynamic exercise in humans. Diabetes 48: 1815-1821.

43. Higaki Y, Hirshman MF, Fujii N, Goodyear LJ (2001) Nitric oxide increase glucose uptake through a mechanism that is distinct from the insulin and contraction pathways in rat skeletal muscle. Diabetes 50: 241-247.

44. Kahn NN, Acharya K, Bhattacharya S, Acharya R, Mazumder S, et al. (2000) Nitric oxide: the "second messenger" of insulin. IUBMB Life 49: 441-450.

45. Evans JL, Maddux BA, Goldfine ID (2005) The molecular basis for oxidative stress-induced insulin resistance. Antioxid Redox Signal 7: 1040-1052.

46. Antonio C, Enrico M (2004) Is Oxidative Stress the Pathogenic Mechanism Underlying Insulin Resistance, Diabetes, and Cardiovascular Disease? The Common Soil Hypothesis Revisited. Arteriosclerosis, Thrombosis, and Vascular Biology 24: 816-823.

47. Surwit RS, Feinglos MN (1988) Stress and autonomic nervous system in type II diabetes. A hypothesis. Diabetes Care 11: 83-85. 